\title{
How Species Affect Each Other's Evolution
}

\author{
Douglas J. Futuyma
}

Published online: 29 January 2010

(C) Springer Science+Business Media, LLC 2010

Charles Darwin may fairly be considered the father not only of evolutionary biology, but of ecology. Throughout On the Origin of Species, the two subjects are virtually inseparable, for, as is well known, Darwin's conception of natural selection, inspired by his reading of Malthus, was rooted in competition for limiting food or other resources. He recognized that competition exists not only among members of the same species, but also among species, and that the growth of populations is limited not only by competition, but by predation, parasitism, and disease as well. "Look at a plant in the midst of its range," he wrote; "why does it not double or quadruple its numbers?... If we wished in imagination to give the plant the power of increasing in number, we should have to give it some advantage over its competitors, or over the animals which preyed on it." But although "it is good thus to try in our imagination to give any form some advantage over another[, p]robably in no single instance should we know what to do so as to succeed."

In those lines, Darwin sketched a major research program of population ecology (accounting for population numbers) and of evolutionary ecology. The evolutionary changes that affect a species' interactions with other species are often considered under the term "coevolution," especially if two or more species each evolve in reaction to the other. The "co" in coevolution, perhaps by subconscious association with the "co" in cooperation, may tempt us to imagine processes of mutual adjustment that lead to harmony in nature. But any such romantic notions are belied by harsh reality, and the leading metaphor in the

D. J. Futuyma $(\bowtie)$

Department of Ecology and Evolution, Stony Brook University,

Stony Brook, NY 11794-5245, USA

e-mail: futuyma@life.bio.sunysb.edu literature of coevolution is not the Elysian Fields, but the arms race.

Granted the importance of mutualistic interactions, Darwin's emphasis on competition has nevertheless been justified by many decades of ecological and evolutionary research. Competition for food, water, light, space, or other limiting resources operates among individuals of both the same and different species of many or most kinds of organisms. There are two ways in which mutations could be advantageous to an individual organism in this situation. One way is to be a better competitor, either by being faster at getting the resource (the early bird gets the worm) or by being better at fighting for it. That is what bacteria and many plants do by secreting toxic chemicals (colicins, allelopathic compounds) that suppress the growth of neighboring bacteria or plants. Perhaps the neatest trick along these lines is practiced by some species of Central American acacias, which harbor colonies of specialized ants that not only sting herbivores, but also kill vines and seedlings near their host plant. Of course, the ants are defending their home against competing plants - but also their food, since the acacia provides special food bodies and nectar to keep its defenders in residence.

The other way to deal with competition is to escape or reduce it by switching to a different, underutilized resource. Darwin saw this clearly: "the more diversified the descendants from any one species become in structure, constitution, and habits, by so much will they be better enabled to seize on many and widely diversified places in the polity of nature," applying to nature the principle of division of labor in human society. This principle of divergence in resource use explains why populations and species become adapted to habitats or resources to which they may at first not be well adapted, for the fitness of "pioneer" genotypes may nevertheless be higher in the new 
context than in the old, where competition is fierce. So it is that we see rapid bursts of diversification, or adaptive radiations, as illustrated by the famous Darwin's finches in the Galápagos Islands, the diverse honeycreepers of the Hawaiian archipelago, and the astonishing numbers of ecologically diverse species of cichlid fishes in the Rift Valley lakes of eastern Africa. Among closely related species of finches or sticklebacks, we can see operating the process that has contributed greatly to the extraordinary ecological diversification of life.

The acacias and their ants to which I have referred are a classic example of a mutualism, a relationship in which each species obtains benefit from the other. (They also exemplify symbiosis, i.e. living together, in contrast to some other mutualisms, such as plant/pollinator interactions.) The provision of benefit is not at all altruistic; it is either payment for service (e.g., paying food for defense) or directly self-serving (the ants protect their home and food supply). Darwin famously wrote that "If it could be proved that any part of the structure of any one species had been formed for the exclusive good of another species, it would annihilate my theory, for such could not have been produced through natural selection." We should notice that Darwin here illustrates the falsifiability of evolutionary theory, which some philosophers have taken to be a characteristic of scientific hypotheses. Moreover, in a world designed by a beneficent intelligent designer, we might expect to see many examples of the interspecific altruism that Darwin here denies. But no one has yet provided the proof that Darwin called for. In fact, the very opposite holds: there are countless cases in which species have evolved to "cheat" on their partners. Species of yucca are pollinated by specialized moths that carefully apply pollen to the stigma of flowers, within which their offspring will feed on the resultant developing seeds. But from these pollinating species, cheating species of yucca moths have evolved several times, that lay their eggs in flowers that the "legitimate" partner has pollinated. Countless species of orchids, the largest family of plants, are deceitful, offering no nectar or other reward to naïve insects; some have even evolved scents that mimic the sex pheromone of a female wasp or bee, and are pollinated by male insects that copulate with the flower. Such exploitation is to be expected of the fundamentally "selfish" nature of natural selection. (We may wonder how an advocate of "intelligent design," i.e. creationism, might explain pseudocopulatory pollination.) One of the major themes in contemporary research on mutualism is how it is maintained despite potential advantages to cheaters. In some cases, the dominant member may punish uncooperative partners; for example, some legumes may reduce the flow of sugars to root nodules with bacteria that do not fix enough nitrogen.
There is a fuzzy border between the reciprocal exploitation that is inherent in mutualisms and the unilateral exploitation that characterizes relationships between parasites and their hosts, predators and their prey, and herbivores and their food plants. The most important thing to understand about these interactions is that there is no necessary reason to think that evolution will tend toward stable coexistence. Predators do not evolve to be prudent and to reduce their predation rate or their reproductive rate so as to insure the persistence of the prey population. Natural selection acts chiefly among individuals within a species population, so individuals that are genetically better at catching prey, and so have a higher rate of survival or reproduction, propagate their genes at a higher rate. The possible future extinction of the prey cannot prevent this process from happening. And we may be sure that predation and parasitism have been responsible for many of the millions of species extinctions during Earth's history, just as they are doing today.

The same is true of parasites, including the microparasites that cause infectious diseases. The difference in this case is that high virulence, or a high rate of parasite reproduction within a host, may cause the death of the individual host before the parasite offspring can disperse to other hosts. In some such cases, a temporary group of parasites - those that inhabit a particular host body - may successfully reproduce or not, depending on the virulence of its members. Thus there can be selection among parasite groups, favoring reduced virulence, while at the same time there is individual selection within each such group for parasite genotypes that reproduce faster and may weaken the host by draining its resources. That is, there may be a conflict between selection at these two levels. Many biological and ecological factors determine which level of selection dominates. After myxoma virus was introduced to control populations of European rabbits that devastated sheep-grazing rangeland in Australia, the virus evolved a somewhat lower level of virulence, apparently because highly virulent virus strains killed their rabbit hosts before mosquitoes could feed and transmit the virus to other rabbits. But in some cases, selection in the parasite population clearly favors high virulence and death of the host. For example, certain viruses proliferate inside caterpillars, including a nuclear polyhedrosis virus that has been used to control populations of gypsy moths in northeastern United States. This virus proliferates so abundantly that it destroys all the tissues, so that the insect virtually melts, dripping countless virus particles into the environment, where they may be ingested by other caterpillars. The more virus offspring are produced, the greater the chance that some will infect new hosts.

Obviously, there is always natural selection of host or prey genotypes that can escape or resist attack. In some 
plants, tolerance of herbivory - the ability to produce many offspring despite having lost tissue - has evolved. The manifold defenses against predators and parasites, ranging from hard shells, to repellent or toxic chemicals, to rapid flight, to the marvellously intricate immune system of vertebrates, account for much of the astonishing diversity of living things, and the adaptations of predators, parasites, and herbivores to counteract these defenses are equally diverse and fascinating. Trypanosomes and some other microbial parasites, for example, can change their coat proteins to evade attack by the host's immune system. Under some conditions, the ongoing selection of prey to evade attack and on predators to capture their food can result in an ongoing escalation of defense and counterdefense that has been likened to an arms race between nations. And like military arms races, such escalation is utterly wasteful from any rational point of view. In a rationally governed world, all nuclear nations would destroy all nuclear arms immediately and simultaneously, and be able to redirect immense sums and resources to more useful ends. So it is with many features of organisms, such as thick shells, that would not require such great investment if ever more proficient natural enemies or competitors had not imposed relentless selection. As Richard Dawkins has pointed out in his recent book The Greatest Show on Earth, tree trunks, which exist only to enable plants to compete with neighbors that otherwise would overtop and shade them, are "standing monuments to futile competition - futile if we think in terms of a planned economy." But the economy of nature is not planned. If it were, the planner would have seen that the great panoply of defenses and counterdefenses is mostly superfluous waste.

But of course natural selection, which is nothing more than differences in survival and reproductive success, cannot plan, and so through the eons, spirals of coevolutionary ploy and counterploy, the evolutionary conflicts between enemies and victims, between competitors, even between mutually exploiting symbionts, have produced waste, evolutionary cul-de-sacs, extinction - but also exquisite forms and functions, adaptations no inventor would ever have conceived, and the "endless forms most beautiful and most wonderful" of which Darwin sang in the last lines of his most wonderful book. 